Marianne Roth, Ursula Enggist \& Claudia Menolfi

Informations du Secrétariat

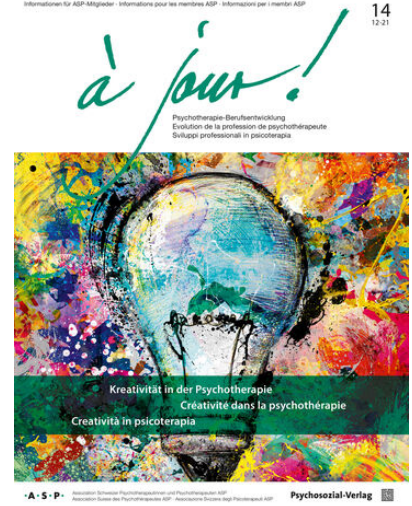

à jour!

Psychotherapie-Berufsentwicklung

7. Jahrgang, Nr. 2, 2021, Seite 46-47

DOI: 10.30820/2504-5199-2021-2-46

Psychosozial-Verlag 


\section{Impressum | Mentions légales}

\author{
à jour! - Psychotherapie-Berufsentwicklung \\ ISSN 2504-5199 (Print-Version) \\ ISSN 2504-5202 (digitale Version) \\ 7. Jahrgang Heft 2/2021, Nr. 14 \\ https://doi.org/10.30820/2504-5199-2021-2
}

\section{Herausgeber}

Assoziation Schweizer Psychotherapeutinnen und Psychotherapeuten ASP

Riedtlistr. 8 | 8006 Zürich | Tel.: 0432689300 | www. psychotherapie.ch

Die Zeitschrift à jour! Psychotherapie-Berufsentwicklung ist ein Informationsorgan der ASP. Gleichzeitig versteht sie sich als Forum ihrer Mitglieder, in dem auch Meinungen geäussert werden, die unabhängig von der Meinung des Vorstandes und der Redaktion sind.

\section{Redaktion}

Peter Schulthess | Redaktionsleitung peter.schulthess@psychotherapie.ch|Tel.: 0765591920

Marianne Roth | marianne.roth@psychotherapie.ch

Veronica Defiébre | veronica.defiebre@psychotherapie.ch

Sandra Feroleto|sandra.feroleto@psychotherapie.ch

\section{Redaktionsschluss}

1. März für Juni-Heft / 15. September für Dezember-Heft

\section{Verlag}

Psychosozial-Verlag

Walltorstr. 10 | D-35390 Gießen | Tel.: +4964196997826

www.psychosozial-verlag.de |info@psychosozial-verlag.de

\section{Abo-Verwaltung | Bezugsgebühren}

Psychosozial-Verlag | bestellung@psychosozial-verlag.de

Jahresabonnement 29,90€ (zzgl. Versand)

Einzelheft 19,90€ (zzgl. Versand)

Studierende erhalten gegen Nachweis $25 \%$ Rabatt.

Das Abonnement verlängert sich um jeweils ein Jahr, sofern nicht eine Abbestellung

bis acht Wochen vor Ende des Bezugszeitrasums erfolgt.

Das Abonnement ist für ASP-Mitglieder im Mitgliedsbeitrag enthalten.

\section{Anzeigen}

Anfragen zu Anzeigen richten Sie bitte an den Verlag (anzeigen@psychosozial-verlag.de) oder die Geschäftsstelle der ASP (asp@psychotherapie.ch).

Es gelten die Preise der auf www. psychosozial-verlag.de einsehbaren Mediadaten. ASP-Mitglieder wenden sich bitte direkt an die Geschäftsstelle der ASP.

Bildnachweise

Titelbild: @ iStock by Getty Images/RapidEye (ID 912441162)

\section{Digitale Version}

Die Zeitschrift à jour! Psychotherapie-Berufsentwicklung ist auch online einsehbar: www.a-jour-asp.ch

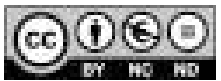

Die Beiträge dieser Zeitschrift sind unter der Creative Commons Attribution-NonCommercial-NoDerivs 3.0 DE Lizenz lizensiert. Diese Lizenz erlaubt die private Nutzung und unveränderte Weitergabe, verbietet jedoch die Bearbeitung und kommerzielle Nutzung. Weitere Informationen finden Sie unter: creativecommons.org/licenses/by-nc-nd/3.0/de

\author{
à jour! - Evolution de la profession de psychothérapeute \\ ISSN 2504-5199 (Version papier) \\ ISSN 2504-5202 (Version numérique) \\ 7. tome numéro 2/2021, 14 \\ https://doi.org/10.30820/2504-5199-2021-2
}

\section{Editeur}

Association Suisse des Psychothérapeutes ASP

Riedtlistr. 8 | 8006 Zürich | Tel.: 0432689300 | www. psychotherapie.ch

La revue à jour! Évolution de la profession du psychothérapeute est un organe d'information de l'ASP. En plus, c'est un forum dans lequel on exprime des avis qui sont indépendants de l'avis du comité et de la rédacion.

\section{Rédaction}

Peter Schulthess | Directeur de rédacion

peter.schulthess@psychotherapie.ch | Tel.: 0765591920

Marianne Roth / marianne.roth@psychotherapie.ch

Veronica Defiébre /veronica.defiebre@psychotherapie.ch

Sandra Feroleto|sandra.feroleto@psychotherapie.ch

\section{Date de rédaction finale}

1er mars pour juin | 15 septembre pour décembre

\section{L'éditeur}

Psychosozial-Verlag

Walltorstr. 10 | D-35390 Gießen | Tel.: +49641969978 26

www.psychosozial-verlag.de|info@psychosozial-verlag.de

\section{Gestion des abonnements | Frais de souscription}

Édition psychosociale | bestellung@psychosozial-verlag.de

Abonnement annuel 29,90€ (frais d'envoi en sus)

Prix du numéro 19,90€ (frais d'envoi en sus)

Les étudiants bénéficient d'une réduction de $25 \%$ sur présentation d'un justificatif.

L'abonnement est reconduit d'un an à chaque fois dans la mesure où aucune résiliation n'a lieu avant le 15 novembre.

L'abonnement est compris dans la cotisation pour les membres ASP.

\section{Annonces}

Veuillez adresser vos demandes de renseignements sur les annonces à l'éditeur (anzeigen@psychosozial-verlag.de) ou au bureau de I'ASP (asp@psychotherapie.ch). Les prix valables sont ceux publiés dans les données médiatiques sur www.psychosozial-verlag.de.

Les membres ASP sont priés de s'adresser directement à la rédaction.

\section{Crédits photographiques}

Couverture: $\odot$ iStock by Getty Images/RapidEye (ID 912441162)

\section{Version numérique}

La revue à jour! Psychothérapie-Développement professionnel est également consultable en ligne : www. a-jour-asp.ch

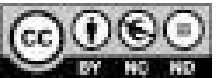

Les articles de cette revue sont disponibles sous la licence Creative Commons 3.0 DE en respectant la paternité des contenus - pas d'utilisation commerciale - sans œuvre dérivée. Cette licence autorise l'utilisation privée et la transmission sans modification, interdit cependant le traitement et l'utilisation commerciale. Veuillez trouver de plus amples informations sous : creativecommons.org/licenses/by-nc-nd/3.0/de 


\title{
Informations du Secrétariat
}

\author{
Marianne Roth | Ursula Enggist | Claudia Menolfi
}

\section{EXCLUSIF À L'ATTENTION DE NOS MEMBRES}

\section{Assemblées des membres}

Les assemblées annuelles des membres ont normalement lieu au printemps et sont annoncées longtemps à l'avance. Les souhaits de points à inscrire à l'ordre du jour peuvent être adressés au secrétariat jusqu’à trois semaines maximum avant la date de la session.

\section{INFORMATIONS IMPORTANTES ET ACTUELLES}

La zone protégée réservée aux membres sur notre site web contient, outre les règlements, des informations importantes destinées à nos membres. Ceci comprend par exemple des documents pour l'assemblée des membres et des actualités sur la crise du coronavirus, le contrat Al ou le paquet all-inclusive de notre agent d'assurance (voir la prochaine colonne), qui est réservé aux membres de I'ASP.

\section{DOCUMENTS ET FORMULAIRES}

\section{Formulaires de facturation pour vos honoraires}

Commandez auprès de notre secrétariat les formulaires pratiques sur lesquels on peut écrire pour vos factures d'honoraires destinées aux patientes et patients, ou retrouvez-les dans la zone protégée réservée aux membres sur notre site web.

\section{Ce que les caisses maladies payent}

Savez-vous quelle caisse maladie rembourse quel montant pour une psychothérapie? Vous trouverez la liste actualisée chaque année dans les "Downloads» sur notre site web, qui est à chaque fois jointe à la première édition à jour! de l’année.

\section{Notices}

Nos fiches informatives contiennent des directives et des informations à l'attention de nos membres. Celles-ci fournissent des renseignements sur :

- la conservation du dossier médical

- le secret professionnel et lobligation de confidentialité

- les abus sexuels

- l'aide aux victimes

- la protection des titres

- les prestations et les tarifs

Les fiches informatives sont réservées à nos membres et sont classées dans la zone protégée réservée aux membres sur notre site web. Ces dernières sont en outre complétées en permanence.

\section{PORTAIL DE RECHERCHE \\ «TROUVER UNE OU UN PSYCHOTHÉRAPEUTE»}

Inscrivez-vous sur le portail de recherche de notre site web avec votre offre de thérapie spécifique. Ce site permet aux personnes à la recherche d'un soutien psychothérapeutique de trouver cette dernière. Si vous le désirez, notre secrétariat vous aidera volontiers à vous inscrire sur le portail de recherche.

\section{ASSURANCES}

\section{Paquet all-inclusive}

Grâce au contrat collectif avec notre agent d'assurance Swiss Quality Broker AG, nous sommes en situation de proposer à nos membres un paquet exclusif de prestations d'assurances économiques qui comprend les offres suivantes :

- Caisse maladie

- Assurance de responsabilité civile professionnelle

- Assurance de protection juridique

- Assurances choses

- Assurance maladie et indemnités journalières en cas de maladie et d'accidents

- Assurance perte de gain

Les membres de l'ASP peuvent en outre bénéficier auprès de ZURICH Assurance de primes économiques pour leurs assurances automobile/moto, habitation, responsabilité civile privée et bâtiment. Informez-vous sur les détails dans la zone protégée réservée aux membres sur notre site web.

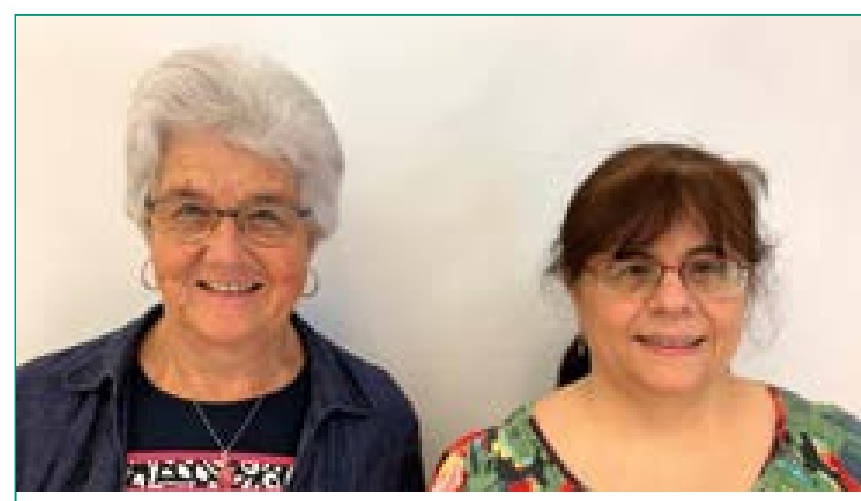

Ursula Enggist ( $\mathrm{g}$ ) et Claudia Menolfi (d) sont vos interlocutrices au secrétariat de l'ASP et se réjouissent des contacts avec nos membres.

Contact direct:

Téléphone: 0432689300

E-mail: asp@psychotherapie.ch 


\section{Contrat Al}

Les psychothérapies destinées aux enfants et aux mineurs, à l'intégration professionnelle ou en cas de difficultés à la naissance peuvent, dans certaines conditions, être remboursées par l'assurance invalidité (AI). Les membres de l'ASP qui sont reconnus au niveau fédéral et disposent d'une autorisation de pratique ont la possibilité d'adhérer au contrat actuel conclu entre l'Office fédéral des assurances sociales (OFAS) et l'ASP. Vous trouverez des informations à ce sujet sur notre site web ou auprès du secrétariat ASP, qui se fera un plaisir d'enregistrer votre demande.

\section{POSSIBILITÉS DE PUBLICITÉ À UN TARIF SPÉCIAL}

\section{Offres d'emplois et locatives}

Vous trouverez dans la rubrique "Actualités» sur notre site web des offres d'emplois à pourvoir et de locaux de cabinets à louer outre des informations de l'association. Avez-vous une offre propre à soumettre ou souhaitez-vous davantage de renseignements sur des appels d’offres? Notre secrétariat vous donnera volontiers davantage de renseignements.

\section{Annonces et annexes}

Utilisez la possibilité de publier vos annonces, par exemple relatives à des manifestations dans nos magazines à jour ! et Psychotherapie-Wissenschaft ou dans notre Newsletter. Les lecteurs sont des psychothérapeutes pratiquant(e)s et des spécialistes de la pratique, de la science et de la recherche. Informez-vous auprès du secrétariat des prix et des parutions ou commandez simplement les données de média.

\section{L'ORGANISATION ASP}

Membres du comité

- Gabriela Rüttimann, présidente

- Veronica Defièbre, vice-présidente

- Sandra Feroleto, délégué de la Suisse française

- Nicola Gianinazzi, délégué de la Suisse italienne

- Kurt Roth

- Peter Schulthess

Secrétariat

- Marianne Roth, directrice

- Ursula Enggist

- Claudia Menolfi

\section{MÉDIAS}

\section{Contact}

Le secrétariat enregistre volontiers les demandes de créateurs de médias. Notre association réunit des praticiennes et praticiens sur différents thèmes - également d'actualité brûlante - en relation à la profession de la psychothérapie.

Bureau médias

Marianne Roth, Téléphone direct:

0432689379

0794202339

marianne.roth@psychotherapie.ch

\section{Possibilités d'expédition}

Nos membres ont la possibilité, de communiquer par circulaire électronique leurs propres manifestations à d'autres membres de l'ASP. Cette offre à des conditions spéciales est exclusivement réservée à nos membres.

\section{Inscription d'agenda sur le site web}

Outre nos propres inscriptions dans l'agenda, nos membres ont la possibilité d'utiliser l' «Agenda» sur le site web pour publier leurs propres manifestations et séminaires actuels et autres événements.

\section{NOS PUBLICATIONS}

\section{Newsletter paraissant tous les trimestres}

Notre Newsletter vous tient au courant des activités actuelles de l'association. Elle contient en outre des informations sur les développements les plus récents aux niveaux professionnel, socio-politique et administratif. Les informations relatives à nos manifestations et séminaires ainsi qu'un mot personnel de notre présidente ne doivent pas manquer. Chaque année voit généralement paraître quatre numéros, qui sont également publiés sur notre site web.

\section{Formations postgrade et séminaires}

Vous trouverez les offres relatives à nos manifestations, formations postgrade et séminaires actuels etc. sur notre site web dans l' «Agenda» ou dans la rubrique «Actualités», sous «Manifestations». 